Supporting Information

\title{
Nanomedicine-cum-Carrier by Co-assembly of Natural Small Products for Synergistic Enhanced Antitumor with Tissues Protective Actions
}

Jiacheng Wang, Haitian Zhao, Wenshu Qiao, Jianjun Cheng, Ying Han, Xin Yang*

School of Chemistry and Chemical Engineering, Harbin Institute of Technology,

No.92 West Dazhi Street, Nan Gang District, Harbin, Heilongjiang, 150001, P.R.China

*Corresponding Author: yangxin@hit.edu.cn 


\section{Experimental Procedures}

Separation, extraction and identification of natural small molecules (NSMs). All

the NSMs used in this study were isolated and purified according to the previous

experience accumulation and laboratory experimental results ${ }^{1-2}$. All NSMs were

analyzed qualitatively and quantitatively by high performance liquid chromatography

(HPLC) and compared with the published data.

\section{Co-assembly morphology of all hybrid NPs}

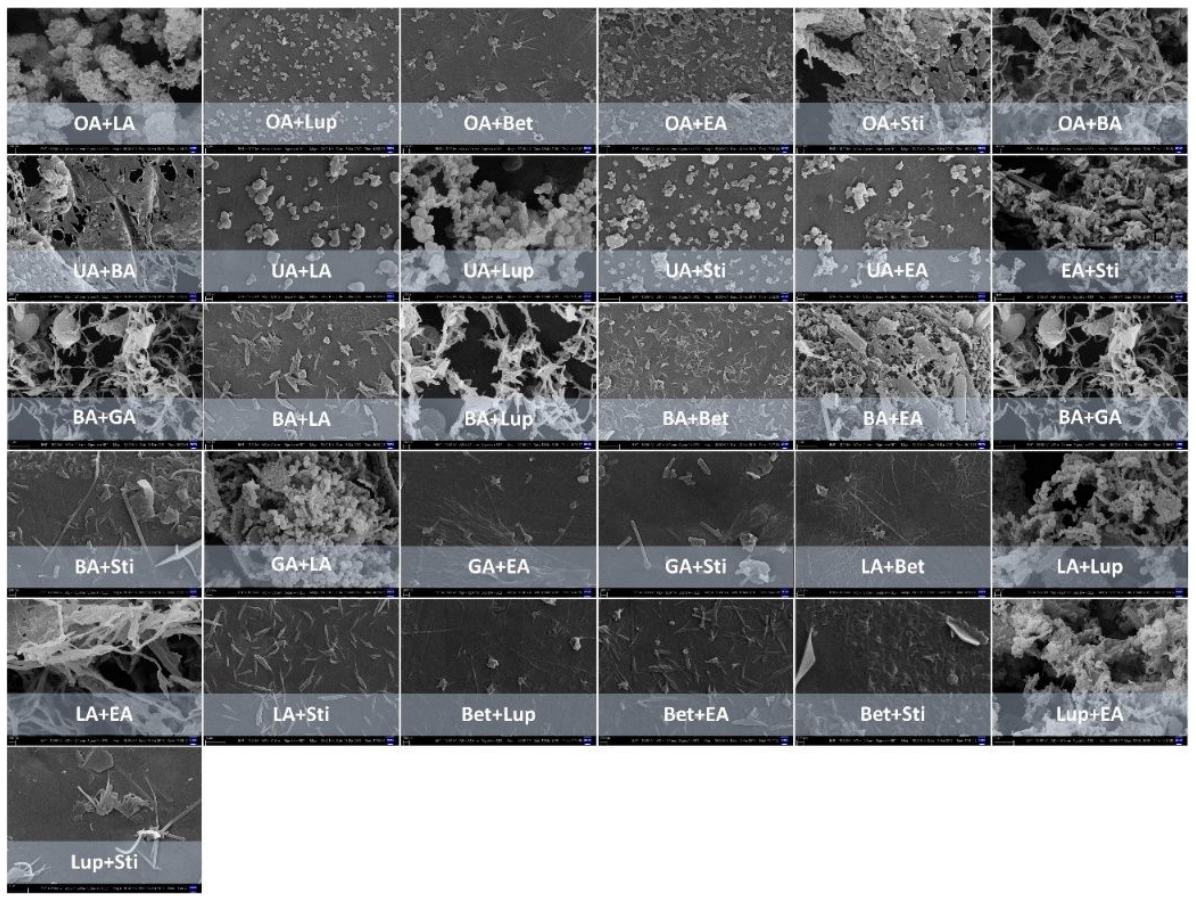

Figure S1 SEM images of hybrid NPs. 


\section{Steric resistance effect}

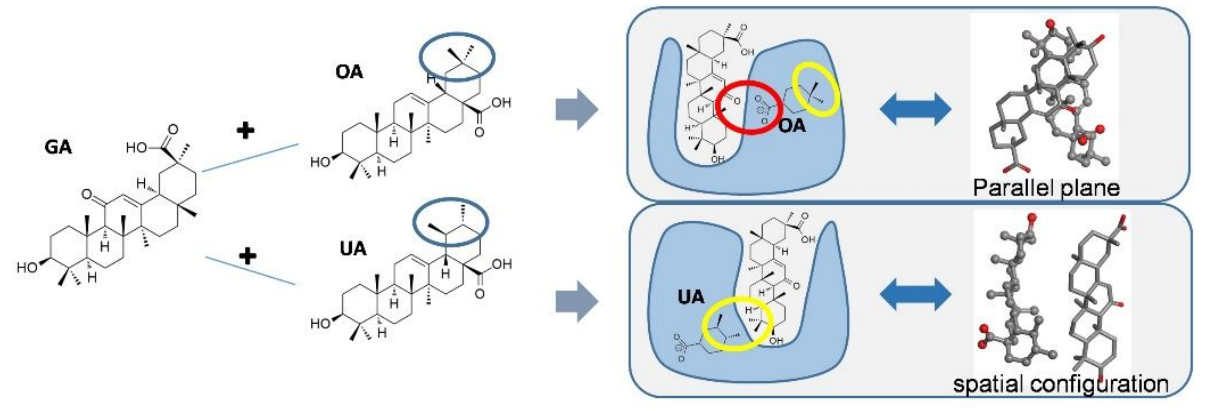

Figure S2 Different methyl positions lead to different results. There is only one methyl site difference between OA and UA, and the results are quite different. OA can be co-assembled with GA, but UA cannot be co-assembled with GA (The blue circle represents the methyl group in two molecular structures, the red circle represents the hydrogen bond, and the yellow circle represents the hydrophobic surface) 
Supplementary data for molecular dynamics simulations (1)
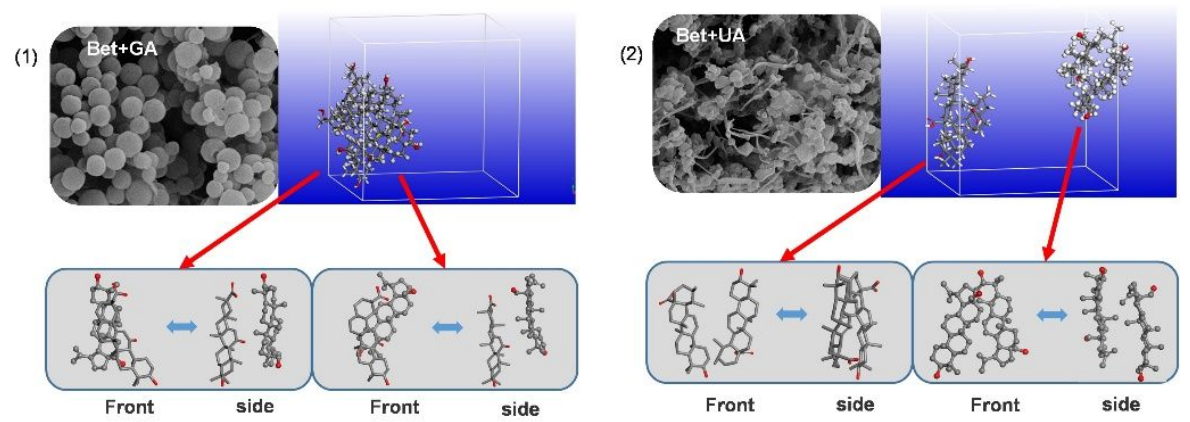

Figure S3 Molecular dynamics simulation of multi molecules. (1) Bet-GA, all molecules interact to form an aggregate (2) Bet+UA, Bet-Bet forms a dimer, and UAUA forms a dimer 
Supplementary data for molecular dynamics simulations (2)

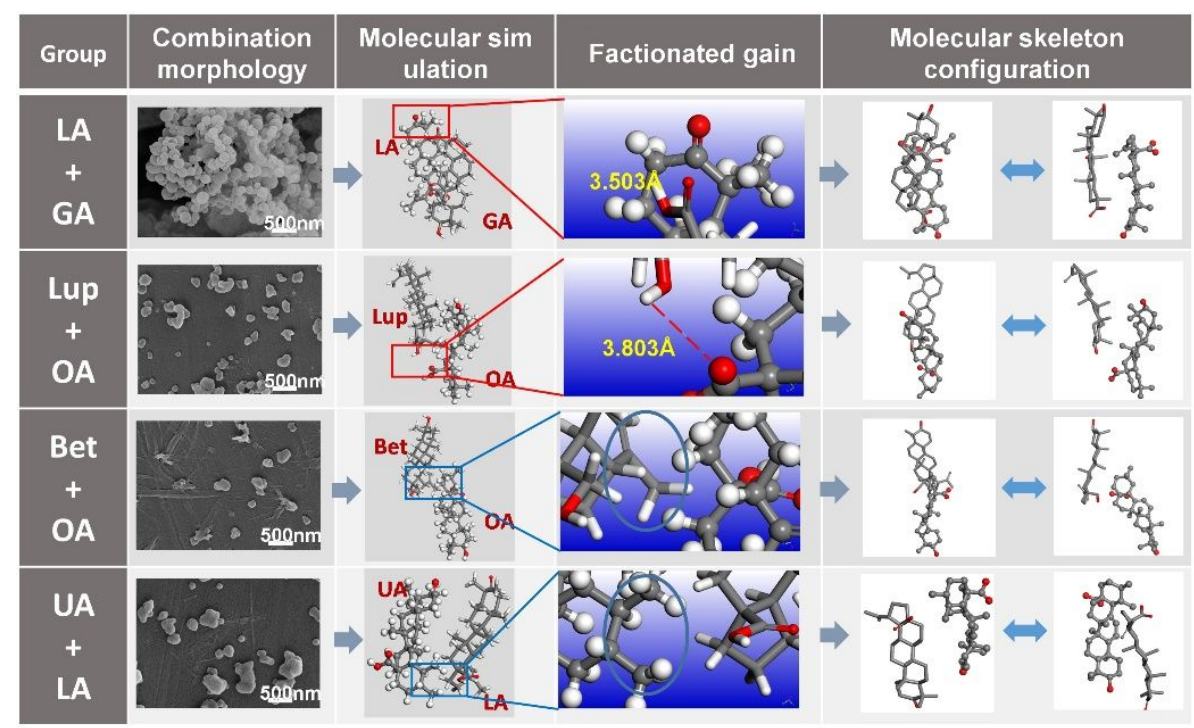

Figure S4 Additional data for molecular dynamics simulation. LA + GA and Lup + OA form co-assembled NPs, and hydrogen bonds can be formed between the two molecules, and the spatial positions of the molecular skeletons of the two molecules are in parallel plane configuration. Bet $+\mathrm{OA}$ and UA + LA did not form coassembled NPs, the two molecules are matched with hydrophobic sides, and the molecular skeleton of the two molecules is in a spatial configuration 
Supplementary data of infrared spectrum

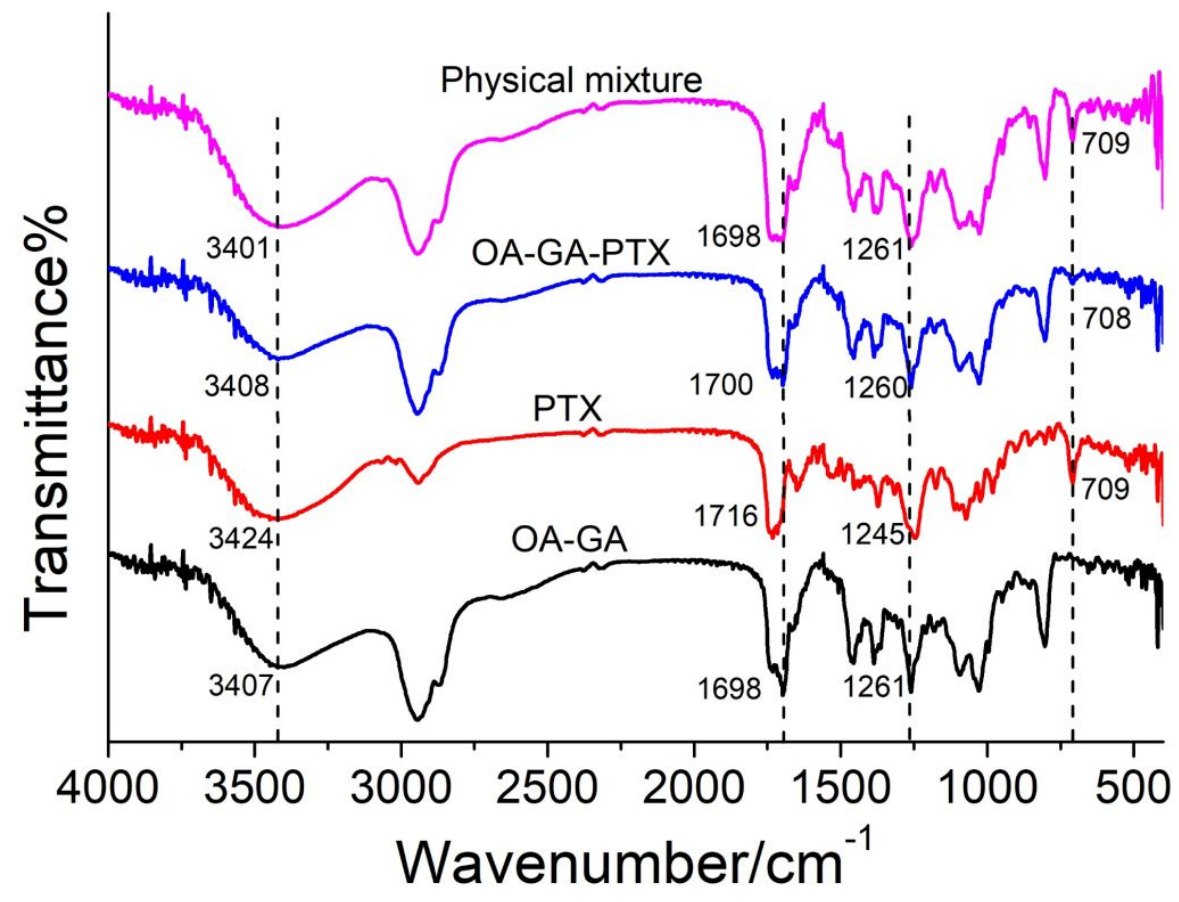

Figure S5 Overlay of FTIR spectra of OA-GA, PTX, OA-GA-PTX and physical mixture of OA-GA and PTX. 
Molecular dynamics simulation of OA GA PTX systems

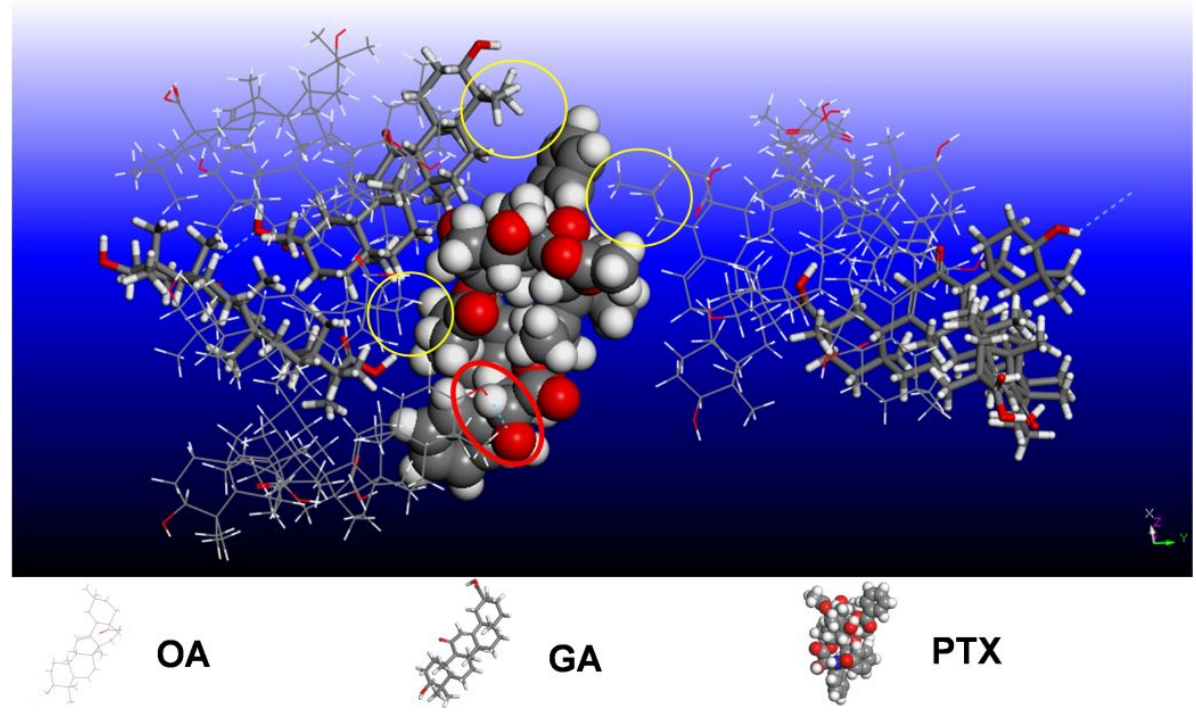

Figure S6 Cluster analysis of OA-GA-PTX systems (yellow circle represents hydrophobic side chain and red circle represents hydrogen bond interaction) 


\section{NPs stability}
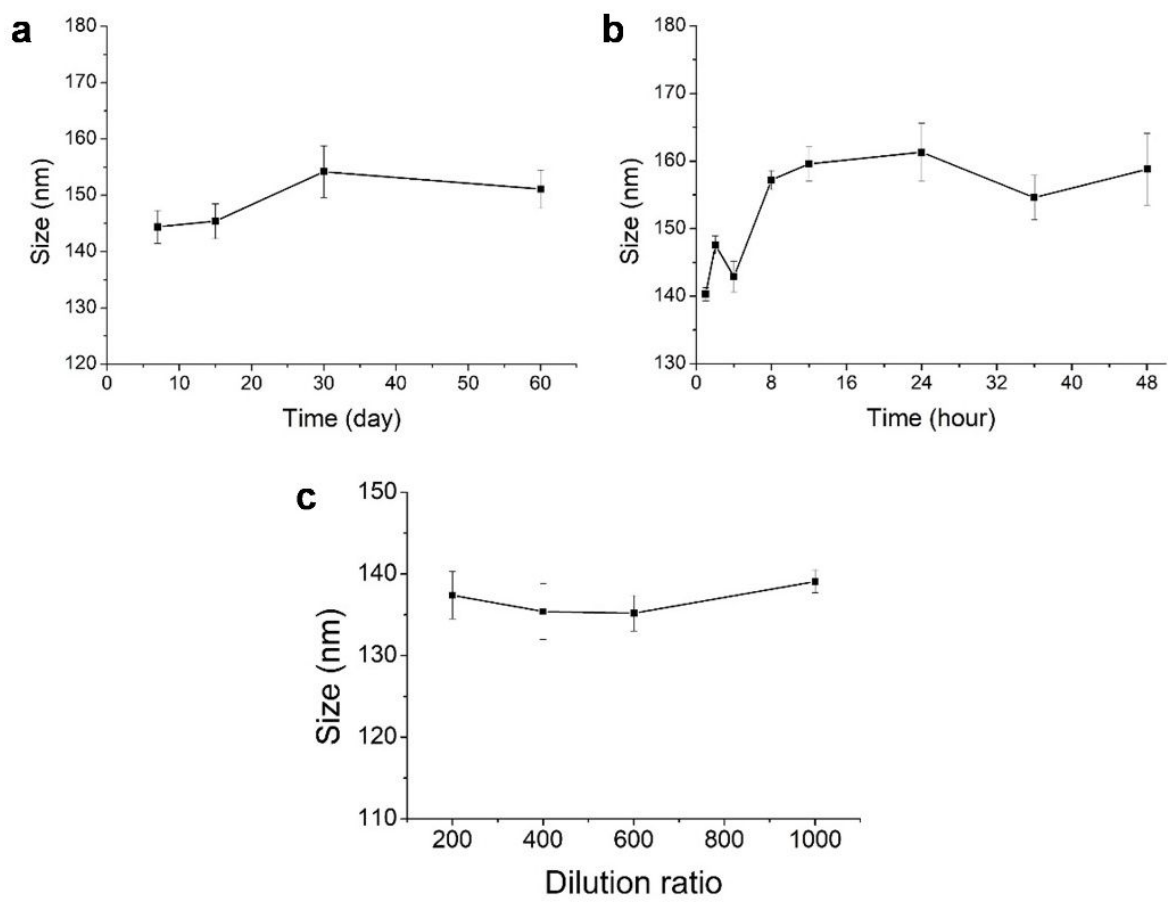

Figure S7 The stability study of OA-GA-PTX NPs. a. The redispersibility and stability of co-assembled NPs under different storage time b. Serum stability of coassembled NPs c. Dilution stability of co-assembled NPs 
Wettability measurement

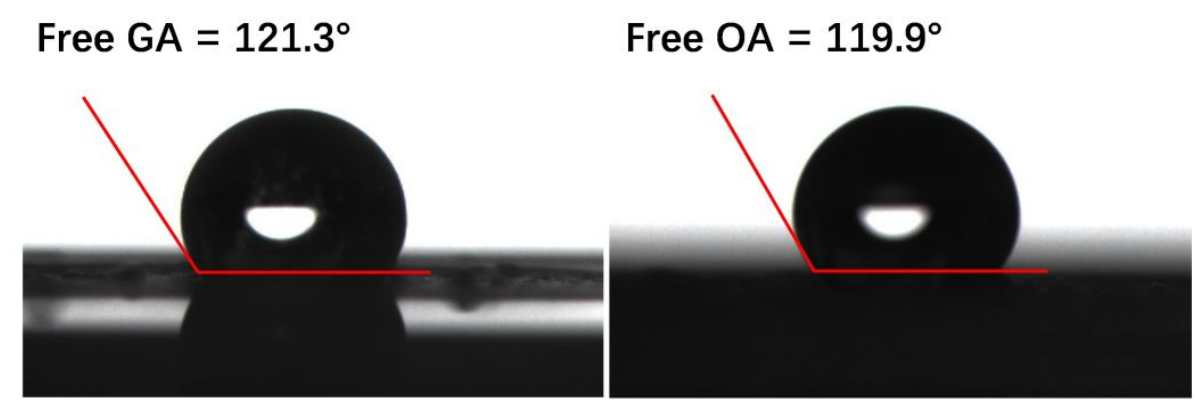

$$
\text { GA NPs }=87.6^{\circ} \quad \text { OA NPs }=72.2^{\circ}
$$

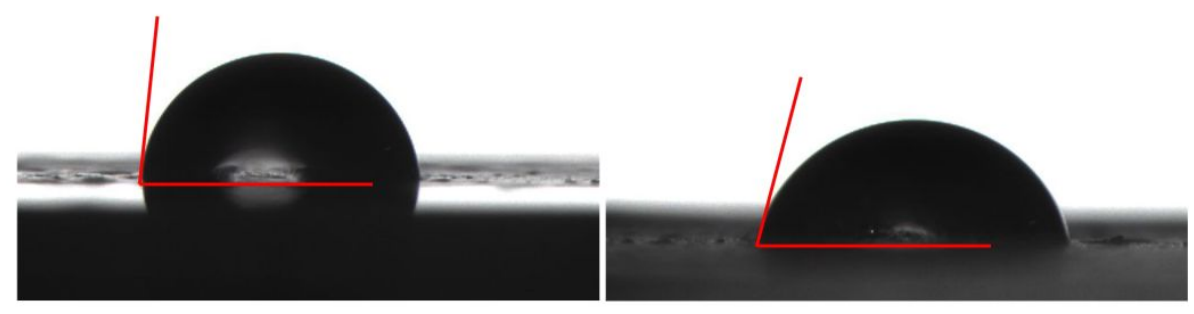

$$
\text { OA-GA NPs }=83.6^{\circ} \quad \text { OA-GA-PTX NPs }=77.2^{\circ}
$$
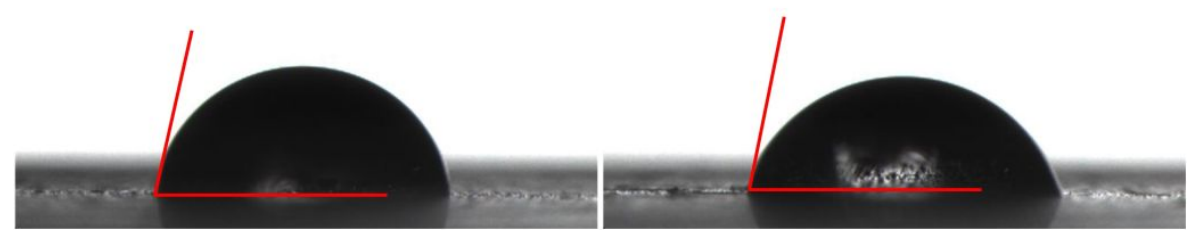

Figure S8 Three-phase contact angles of free natural products and nano preparations. 
Post-infusion serum drug concentration versus time curve

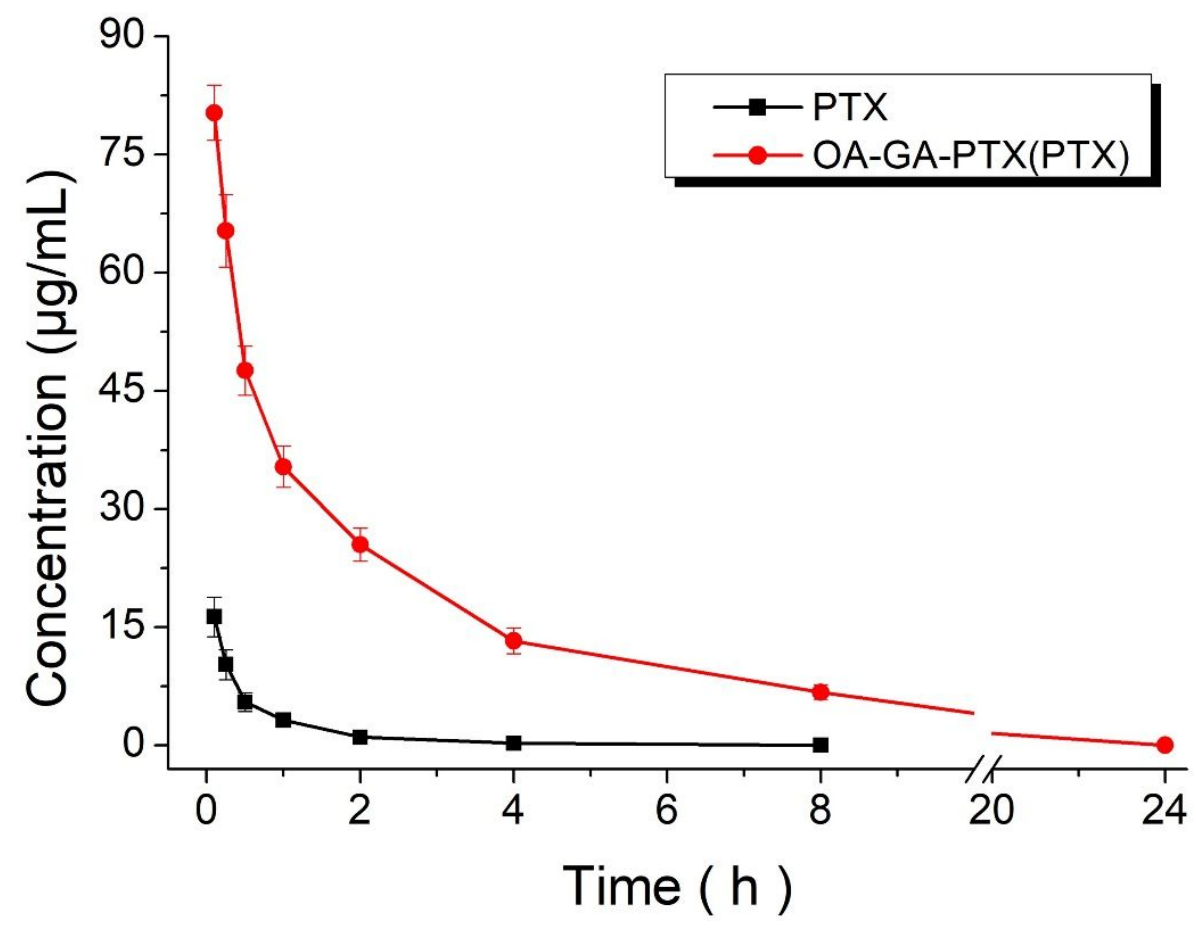

Figure S9 Post-infusion serum drug concentration versus time curve. PTX: free paclitaxel; OA-GA-PTX (PTX): Paclitaxel in OA-GA-PTX NPs. Mean \pm S.D. $(n=3)$ 


\section{Supplementary data for in vitro anti-tumor experiments}

a
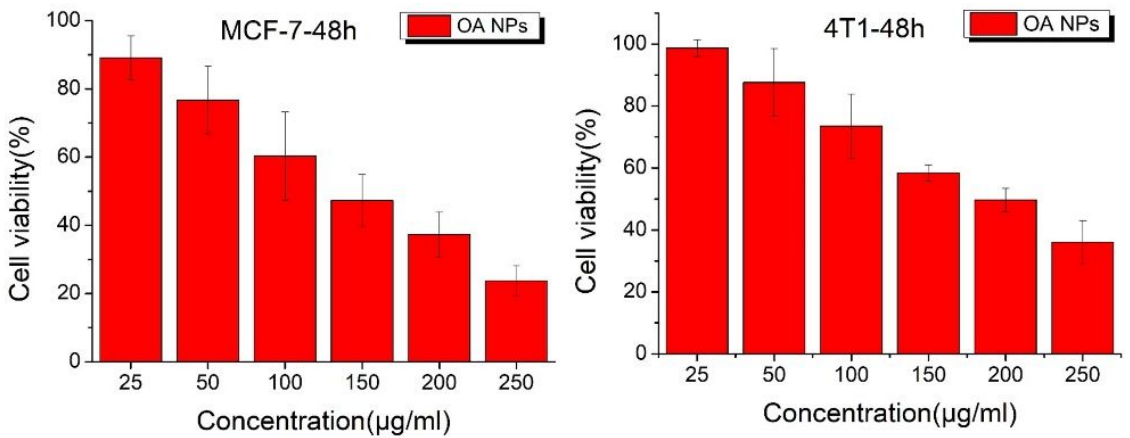

b
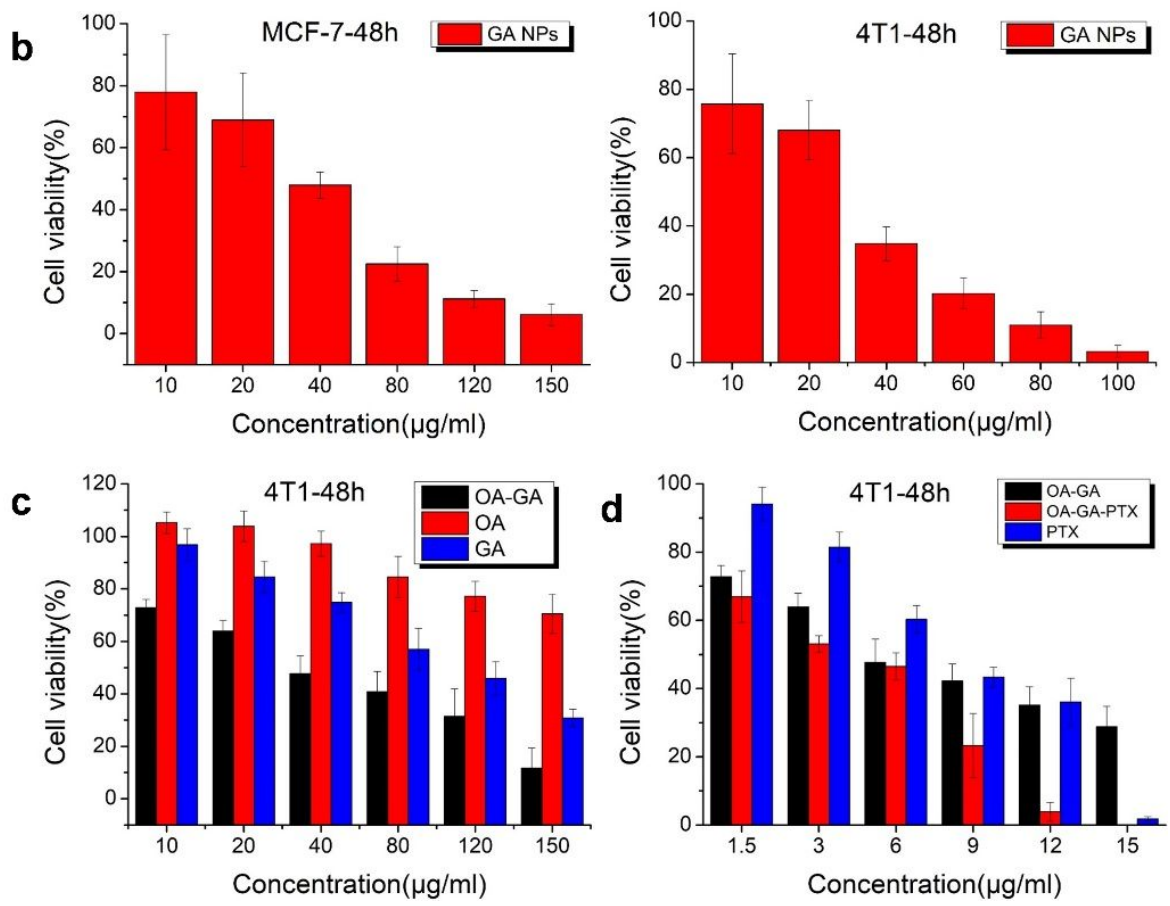

Figure S10 Supplementary data for in vitro cytotoxicity experiment. a.

Cytotoxicity test of OA NPs on MCF-7 cells and 4T1 cells b. Cytotoxicity test of GA

NPs on MCF-7 cells and 4T1 cells c. The growth inhibitory effects of OA, GA and OA-

GA NPs on 4T1 cells for 48h d. The growth inhibitory effects of PTX, OA-GA and OA-GA-PTX on $4 \mathrm{~T} 1$ cells for $48 \mathrm{~h}$ 
Histogram of tumor inhibition rate

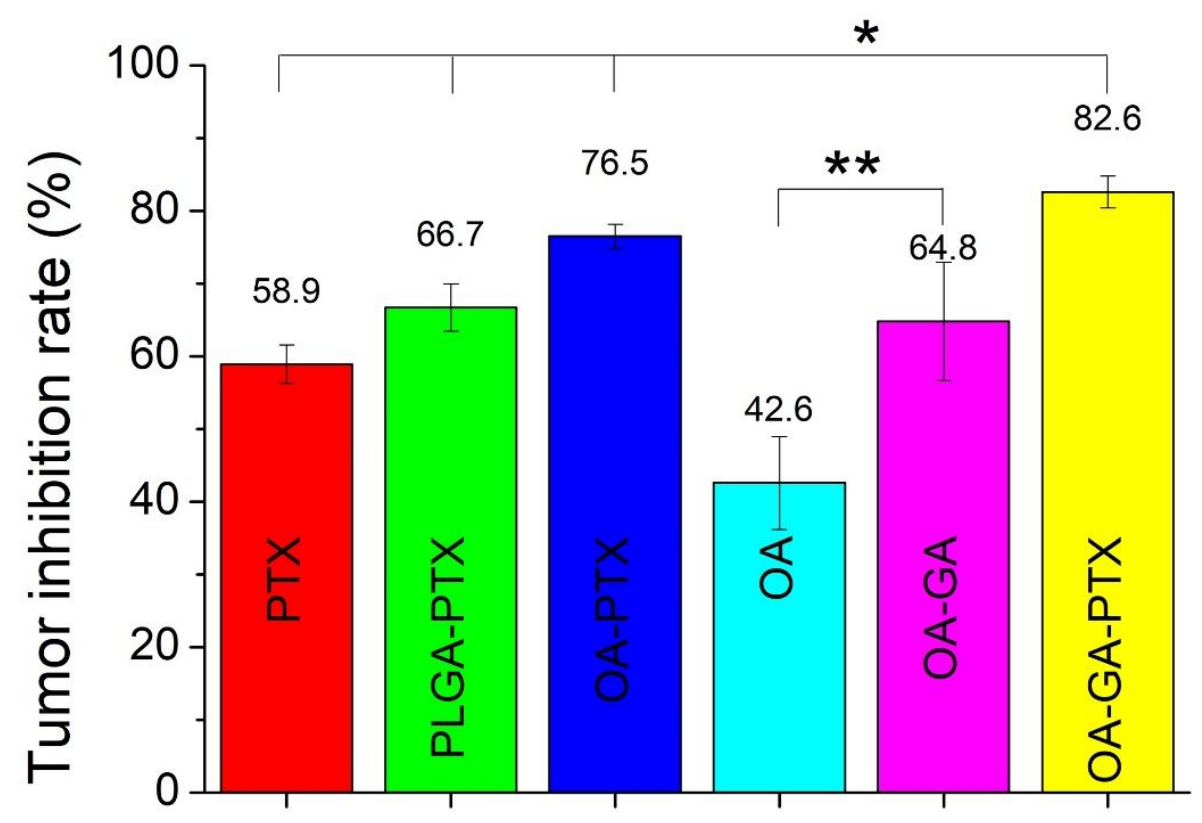

Figure S11 Comparison of tumor volume inhibition rates in different treatment groups. Compared with the PTX and PLGA-PTX treatment groups, the tumor volume inhibition rate in the OA-GA-PTX NPs treatment group was significantly enhanced. $* \mathrm{P}<0.05, * * \mathrm{P}<0.01$ 


\section{Biochemical index of blood}
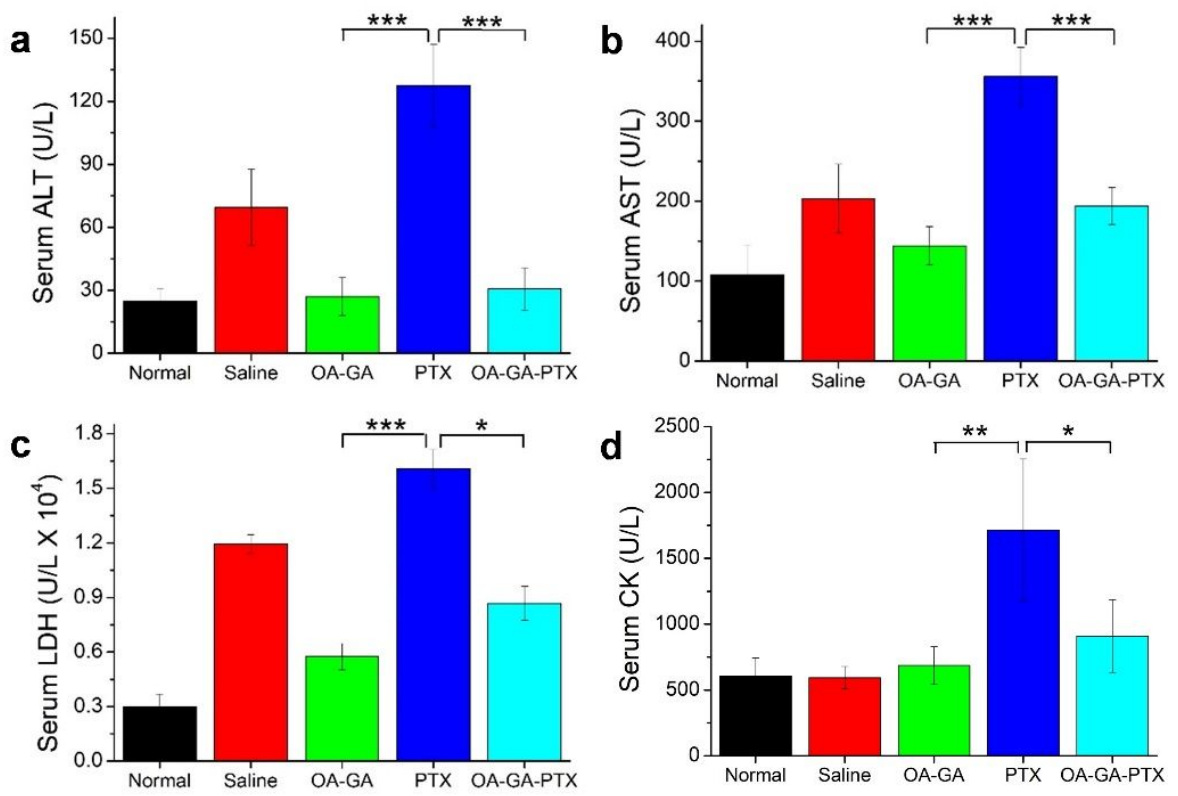

Figure S12 Comparisons of blood biochemical indexes. a. The levels of ALT in the serum b. The levels of AST in the serum c. The levels of LDH in the serum d. The levels of $\mathrm{CK}$ in the serum 
Representative H\&E staining of major organs

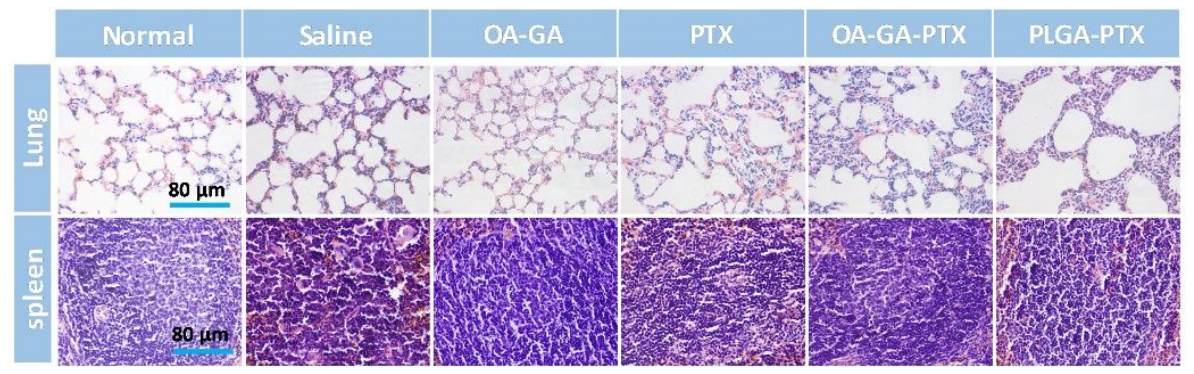

Figure S13 Representative H\&E staining of major organs of healthy mice and 4T1

breast cancer tumor-bearing mice. No obvious morphological changes were observed in lung and spleen tissue 


\begin{tabular}{|c|c|c|c|c|c|}
\hline $\begin{array}{c}\text { Formula } \\
\text { Abbreviation }\end{array}$ & Diamater $(\mathbf{n m})$ & PDI & $\begin{array}{c}\text { Formula } \\
\text { Abbreviation }\end{array}$ & Diamater $(\mathbf{n m})$ & PDI \\
\hline OA & $\mathbf{1 7 3 \pm 2}$ & $\mathbf{0 . 0 5} \pm \mathbf{0 . 0 2}$ & BS & $356 \pm 7$ & $0.31 \pm 0.06$ \\
\hline UA & $\mathbf{1 9 5} \pm \mathbf{4}$ & $\mathbf{0 . 0 5} \pm \mathbf{0 . 0 1}$ & EA & $486 \pm 12$ & $0.35 \pm 0.04$ \\
\hline BA & $1661 \pm 141$ & $0.69 \pm 0.12$ & MA & $297 \pm 3$ & $0.10 \pm 0.02$ \\
\hline GA & $557 \pm 13$ & $0.39 \pm 0.04$ & Lup & $339 \pm 14$ & $0.26 \pm 0.05$ \\
\hline LA & $877 \pm 19$ & $0.69 \pm 0.05$ & Erg & $484 \pm 62$ & $0.51 \pm 0.11$ \\
\hline Bet & $671 \pm 14$ & $0.43 \pm 0.02$ & Sti & $500 \pm 41$ & $0.44 \pm 0.04$ \\
\hline
\end{tabular}

Table S1 NPs diameters and polydispersity index (PDI). 


\begin{tabular}{|c|c|c|c|c|c|}
\hline $\begin{array}{c}\text { Formula } \\
\text { Abbreviation }\end{array}$ & Diamater (nm) & PDI & $\begin{array}{c}\text { Formula } \\
\text { Abbreviation }\end{array}$ & Diamater (nm) & PDI \\
\hline $\mathrm{OA}+\mathrm{BA}$ & $346 \pm 4$ & $0.16 \pm 0.03$ & $B A+S t i$ & $658 \pm 66$ & $0.44 \pm 0.10$ \\
\hline $\mathrm{OA}+\mathrm{GA}$ & $149 \pm 12$ & $0.05 \pm 0.02$ & $G A+L A$ & $232 \pm 1$ & $0.04 \pm 0.02$ \\
\hline$O A+L A$ & $327 \pm 5$ & $0.11 \pm 0.01$ & GA+Bet & $278.3 \pm 1$ & $0.09 \pm 0.03$ \\
\hline $\mathrm{OA}+\mathrm{Bet}$ & $480 \pm 6$ & $0.38 \pm .01$ & $\mathrm{GA}+\mathrm{BS}$ & $1996 \pm 134$ & 1 \\
\hline $\mathrm{OA}+\mathrm{BS}$ & $308 \pm 4$ & $0.15 \pm 0.02$ & GA+Lup & $267 \pm 4$ & $0.22 \pm 0.02$ \\
\hline OA+Lup & $270 \pm 1$ & $0.12 \pm 0.05$ & $\mathrm{GA}+\mathrm{Erg}$ & $791 \pm 84$ & $0.63 \pm 0.03$ \\
\hline $\mathrm{OA}+\mathrm{Erg}$ & $433 \pm 15$ & $0.34 \pm 0.08$ & $\mathrm{GA}+\mathrm{Sti}$ & $582 \pm 4$ & $0.43 \pm 0.06$ \\
\hline $\mathrm{OA}+\mathrm{Sti}$ & $300 \pm 3$ & $0.16 \pm 0.02$ & $L A+B e t$ & $713 \pm 31$ & $0.44 \pm 0.06$ \\
\hline$U A+B A$ & $349 \pm 2$ & $0.09 \pm 0.01$ & $\mathrm{LA}+\mathrm{BS}$ & $622 \pm 47$ & $0.44 \pm 0.03$ \\
\hline$U A+G A$ & $402 \pm 8$ & $0.22 \pm 0.02$ & LA+Lup & $238 \pm 2$ & $0.11 \pm 0.01$ \\
\hline$U A+L A$ & $374 \pm 1$ & $0.20 \pm 0.01$ & $L A+E r g$ & $584 \pm 15$ & $0.32 \pm 0.04$ \\
\hline $\mathrm{UA}+\mathrm{Bet}$ & $371 \pm 7$ & $0.14 \pm 0.01$ & $\mathrm{LA}+\mathrm{Sti}$ & $544 \pm 10$ & $0.18 \pm 0.02$ \\
\hline$U A+B S$ & $420 \pm 5$ & $0.18 \pm 0.01$ & Bet+BS & $604 \pm 67$ & $0.43 \pm 0.08$ \\
\hline UA+Lup & $508 \pm 12$ & $0.41 \pm 0.05$ & Bet+Lup & $1217 \pm 159$ & $0.63 \pm 0.12$ \\
\hline $\mathrm{UA}+\mathrm{Erg}$ & $476 \pm 3$ & $0.25 \pm 0.01$ & Bet+Erg & $426 \pm 6$ & $0.23 \pm 0.01$ \\
\hline$U A+S t i$ & $376 \pm 2$ & $0.11 \pm 0.05$ & Bet+Sti & $629 \pm 39$ & $0.51 \pm 0.10$ \\
\hline$B A+G A$ & $565 \pm 33$ & $0.41 \pm 0.08$ & BS+Lup & $294 \pm 2$ & $0.20 \pm 0.02$ \\
\hline$B A+L A$ & $354 \pm 5$ & $0.22 \pm 0.02$ & $\mathrm{BS}+\mathrm{Erg}$ & $490 \pm 155$ & $0.45 \pm 0.17$ \\
\hline $\mathrm{BA}+\mathrm{Bet}$ & $501 \pm 4$ & $0.21 \pm 0.03$ & $\mathrm{BS}+\mathrm{Sti}$ & $418 \pm 46$ & $0.34 \pm 0.27$ \\
\hline$B A+B S$ & $605 \pm 6$ & $0.28 \pm 0.02$ & Lup+Erg & $290 \pm 11$ & $0.28 \pm 0.01$ \\
\hline BA+Lup & $401 \pm 9$ & $0.20 \pm 0.03$ & Lup+Sti & $347 \pm 47$ & $0.31 \pm 0.12$ \\
\hline $\mathrm{BA}+\mathrm{Erg}$ & $514 \pm 57$ & $0.49 \pm 0.11$ & Erg+Sti & $437 \pm 9$ & $0.36 \pm 0.01$ \\
\hline
\end{tabular}

Table S2 Hybrid Nanoparticle diameters and polydispersity index (PDI). 


\begin{tabular}{|c|c|c|c|}
$\begin{array}{c}\text { Formula } \\
\text { Abbreviation }\end{array}$ & Diameter $(\mathrm{nm})$ & PDI & $\begin{array}{c}\text { Zeta potential } \\
(\mathrm{mV})\end{array}$ \\
\hline OA & $173 \pm 2$ & $0.05 \pm 0.02$ & $-20.5 \pm 1.6$ \\
\hline GA & $557 \pm 13$ & $0.29 \pm 0.04$ & $-12.2 \pm 0.2$ \\
\hline OA-GA & $149 \pm 12$ & $\mathbf{0 . 0 5} \pm \mathbf{0 . 0 2}$ & $-22.9 \pm 1.6$ \\
\hline Data are presented as mean $\pm \mathbf{s . d .}(n=3)$ & \\
\hline
\end{tabular}

Table S3 Size and Zeta potential of OA, GA, OA-GA after being prepared into NPs. OA NPs and OA-GA NPs have a uniform diameter distribution; GA NPs diameters are larger than $500 \mathrm{~nm}$ 


\begin{tabular}{|c|c|c|c|}
\hline $\begin{array}{c}\text { Formula } \\
\text { Abbreviation }\end{array}$ & Particle Diameter $(\mathrm{nm})$ & PDI & Zeta potential $(\mathrm{mV})$ \\
\hline $\mathrm{OA:GA}=1: 9$ & $342 \pm 36$ & $0.390 \pm 0.03$ & $-16.6 \pm 0.5$ \\
\hline $\mathrm{OA:GA}=2: 8$ & $348 \pm 20$ & $0.265 \pm 0.01$ & $-16.4 \pm 1.3$ \\
\hline $\mathrm{OA}: \mathrm{GA}=4: 6$ & $163 \pm 13$ & $0.165 \pm 0.02$ & $-19.7 \pm 2.1$ \\
\hline OA:GA=5:5 & $147 \pm 10$ & $0.067 \pm 0.04$ & $-22.4 \pm 0.6$ \\
\hline OA:GA=6:4 & $154 \pm 11$ & $0.060 \pm 0.01$ & $-21.8 \pm 1.0$ \\
\hline OA:GA=7:3 & $154 \pm 12$ & $0.063 \pm 0.03$ & $-19.9 \pm 0.6$ \\
\hline OA:GA=8:2 & $160 \pm 8$ & $0.048 \pm 0.04$ & $-19.3 \pm 0.2$ \\
\hline OA:GA=9:1 & $166 \pm 14$ & $0.102 \pm 0.03$ & $-19.5 \pm 0.7$ \\
\hline Data are presented as mean \pm s.d. $(n=3)$ & & \\
\hline
\end{tabular}

Table S4 Diameters, polydispersity index and Zeta potential of OA-GA NPs with varied mixed ratio. 


\begin{tabular}{|c|c|c|c|c|}
$\begin{array}{c}\text { Formula } \\
\text { abbreviation }\end{array}$ & $\begin{array}{c}\text { Diameter } \\
(\mathrm{nm})\end{array}$ & PDI & $\begin{array}{c}\text { Zeta potential } \\
(\mathrm{mV})\end{array}$ & $\begin{array}{c}\text { Loading } \\
\text { Capacity(\%) }\end{array}$ \\
\hline PTX & $2968 \pm 190$ & $0.75 \pm 0.05$ & $-7.3 \pm 0.5$ & - \\
\hline OA-GA & $149 \pm 12$ & $0.05 \pm 0.02$ & $-22.9 \pm 1.6$ & - \\
\hline OA-GA-PTX & $138 \pm 7$ & $0.09 \pm 0.02$ & $-22.4 \pm 1.8$ & $15.1 \pm 0.4$ \\
\hline
\end{tabular}

Table S5 Characterization of PTX, OA-GA, OA-GA-PTX NPs by DLS. 


\section{References}

(1) Zhi, K.; Zhao, H.; Yang, X.; Zhang, H.; Wang, J.; Wang, Z. Solvent-Induced

Gel Formation Hypothesis for Natural Product Gelators with Polycyclic Structures. ChemPlusChem 2018, 83 (8), 797-803.

(2) Zhi, K.; Zhao, H.; Yang, X.; Zhang, H.; Wang, J.; Wang, J.; Regenstein, J. M. Natural Product Gelators and a General Method for Obtaining Them from Organisms. Nanoscale 2018, $10(8), 3639-3643$. 